Artículo

\title{
El proceso de comercialización del café en el sur del Estado de México
}

\author{
Felipe de Jesús González Razo ${ }^{1 \S}$ \\ Dora Ma. Sangerman-Jarquín ${ }^{2}$ \\ Samuel Rebollar Rebollar ${ }^{3}$ \\ José Miguel Omaña Silvestre ${ }^{4}$ \\ Juvencio Hernández Martínez ${ }^{5}$ \\ José Luis Morales Hernández ${ }^{1}$
}

${ }^{1}$ Unidad Académica Profesional Tejupilco-Universidad Autónoma del Estado de México. C. Leopoldo Flores Valdés s/n, Col. Rincón de Aguirre, Tejupilco, México. CP. 51412. Tel. 724 2675422, ext. 124. (joselui2001@hotmail.com). ${ }^{2}$ Campo Experimental Valle de México-INIFAP. Carretera Los ReyesTexcoco km 13.5, Coatlinchán, Texcoco, Estado de México. CP. 56250. Tel. 018000882222 , ext. 85353. (sangerman.dora@inifap.gob.mx). ${ }^{3}$ Centro Universitario UAEM-Temascaltepec. Carretera Toluca Tejupilco km 67.5, Barrio de Santiago, Temascaltepec, México. CP. 51300. (samrere@hotmail.com). ${ }^{4}$ Colegio de Postgraduados. Carretera México-Texcoco km 36.5, Montecillo, Texcoco, Estado de México. CP. 56230. (miguelom@colpos.mx). ${ }^{5}$ Centro Universitario UAEM Texcoco. Av. Jardín Zumpango s/n, El Tejocote, Texcoco, México. CP. 56259. (jh_martinez1214@yahoo.com.mx).

${ }^{\S}$ Autor para correspondencia: fegora24@yahoo.com.mx.

\section{Resumen}

El presente estudio analiza el sistema de producción predominante en la explotación de café (Coffea arabica L.) en el sur del Estado de México, su proceso de comercialización y el impacto económico que genera dicha actividad entre los principales agentes participantes, el año de referencia de la investigación fue 2018. Se determinó el sistema de producción preponderante en la región, se identificaron los principales canales de comercialización que sigue el producto desde su salida en la finca hasta su arribo al consumidor final y se calcularon, a precios corrientes, los márgenes de comercialización resultantes durante todo el proceso. El aprovechamiento del café en el sur del Estado de México se desarrolla bajo un sistema de producción rústico o de montaña, de sombra, el cual se desarrolla en pequeñas unidades de producción dispersas, como un complemento de otras actividades agropecuarias. El canal de comercialización tradicional empleado para llevar el producto desde la explotación hasta el consumidor final fue: la venta directa del productor a la cooperativa de productores, la cual le añade valor al producto y opera como principal intermediario. La participación de los productores en el precio final del producto fue en promedio de $75.46 \%$, el acopiador participó con $15.67 \%$ y los detallistas $8.92 \%$. El margen de comercialización total promedio $63.00 \$ \mathrm{~kg}^{-1}$, del cual la cooperativa consiguió el mayor margen promedio con $23.95 \$$ $\mathrm{kg}^{-1}$, mientras el restante $12.65 \$ \mathrm{~kg}^{-1}$ se lo adjudicaron los detallistas.

Palabras clave: Coffea arabica L., comercialización, márgenes de comercialización.

Recibido: mayo de 2019

Aceptado: agosto de 2019 


\section{Introducción}

Hoy en día, México se ubica como un importante productor de café en el mundo, con más de 200 años de tradición, que señala de alguna manera, la importancia que tiene este aromático en la economía agrícola del país, el café que se produce es tipo arábica en diversas variedades, cada una de las cuales guarda una relación con las diferentes regiones ecológicas del país (ASERCA, 1997).

México, considerado tradicionalmente como un productor de café de menor calidad que otros países, tiene no sólo las cualidades idóneas, sino una vocación natural para la producción de café de especialidad sobre todo si tomamos en cuenta que: a) nuestra producción es predominantemente de café de sombra y orgánico, por el resguardo que brindan las selvas, ecosistema donde se cultiva la mayor cantidad de café en nuestro país; b) el 92\% de los productores de café en México poseen superficies menores a 5 ha; y c) al no disponer de grandes cantidades de capital para la inversión como refieren investigaciones de Escamilla y Díaz y Escamilla (2016a y 2016b), se reduce el uso de variedades híbridas y de agroquímicos, haciendo con ello un manejo de producto muy cercano al interés demostrado en el mundo por el consumo de productos alimenticios sanos.

Estos factores, que durante un tiempo fueron vistos como debilidades del sector cafetalero mexicano, permiten otorgar un valor agregado per-se al café mexicano, accediendo a que grandes sectores de la producción de café en nuestro país, estén en posibilidades de ser consolidados y por tanto reconocidos como productores de café de especialidad, obteniendo con ello el beneficio que eso implica (González, 2004; Cruz et al., 2015).

En este sentido, la nueva visión reconoce la relación entre calidad y consumo, de modo que el café es visto como un producto diferenciado, en un mercado segmentado, que cada día demanda mayor calidad; de esta manera, la calidad en cada punto de la cadena de comercialización del café puede ser la respuesta para mejorar el ingreso de los diferentes agentes (ASERCA, 2002; ICO, 2014).

De acuerdo con la Organización Internacional del Café (ICO, 2018), durante la cosecha 2016-2017; $73.5 \%$ de la producción mundial de café se concentró en cinco países: Brasil (35.74\%), Vietnam (16.57\%), Colombia (9.42\%), Indonesia (7.47\%) y Etiopía (4.29\%), por su parte, México se ubicó en la décimo primera posición, con una participación de $2.01 \%$ de la producción mundial (3.1 millones de sacos), esto es, $11.29 \%$ más respecto a la cosecha del periodo anterior.

Respecto al comercio internacional del café, durante la cosecha 2015-2016, 78.93\% de la producción mundial del aromático se comercializó en los mercados del mundo; en este sentido, las exportaciones totales crecieron a una tasa promedio anual 3.1\% en la última década (2005-20062015-2016), con un volumen promedio de 103.6 millones de sacos; cinco países participaron en conjunto con 74.82\% del volumen total exportado en el ciclo 2015-2016: Brasil (30.87\%), Vietnam (22.10\%), Colombia (10.28\%), Indonesia (6.67\%) y Honduras $(4.90 \%)$, por su parte México se ubicó en la onceava posición con 2.3 millones de sacos, $1.93 \%$ de las exportaciones mundiales.

En el país, las regiones cafetaleras se concentran en cuatro zonas: las vertientes del Golfo de México y del Océano Pacífico, la región Centro Norte y la del Soconusco en Chiapas, las cuales abarcan 398 municipios en todos los estados productores (CEFP, 2001; INIFAP, 2013). Asimismo, se dedican a la cafeticultura aproximadamente 283000 productores, que genera 300000 empleos 
temporales, así como 100000 empleos en tareas relacionadas con la agroindustria y comercialización; en este sentido, el aromático representa la fuente de ingresos para más de 70000 familias, que dependen alrededor de 3 millones de personas, consolidándose como un producto estratégico en la generación de empleo, ingreso y desarrollo rural (Leme y Machado, 2013).

La población productora de café, así como la mayor superficie se concentra, en pequeñas unidades de producción; el tamaño promedio de los predios es de 2.7 ha, mientras que $92 \%$ de los cafeticultores del país cuentan con superficies de 5 ha o menos, este hecho que aparentemente es factor de fragilidad por el grado de fragmentación de los predios, representa una ventaja, sobre todo si consideramos el cuidado de tipo artesanal que se da a la producción, lo que permite proporcionar un valor agregado al producto (ASERCA, 2002).

Asimismo, de acuerdo con estadísticas de la SAGARPA (2017), durante el ciclo de producción 2014 se produjeron 1.16 millones de $\mathrm{t}$ de café verde, esto es $7.31 \%$ menos que la producción del ciclo anterior; el cultivo, se centraliza en cuatro entidades federativas, las cuales concentraron $88.72 \%$ de la producción nacional: Chiapas participó con $34.48 \%$, Veracruz $(30.33 \%)$, Puebla (12.77\%) y Oaxaca (11.13\%). Cabe resaltar, que durante el periodo 2000-2014 la producción nacional de café se contrajo, al registrar una tasa de crecimiento promedio anual negativa de 3.19\%, atribuible, principalmente, a problemas de tipo fitosanitario.

Por su parte, el Estado de México se ubicó como el doceavo productor durante 2014, con 427 t (el $0.04 \%$ del total nacional), dicho volumen representó $0.8 \%$ más de la producción registrada el año anterior y apenas $18.57 \%$ del volumen máximo alcanzado en 2003, cuando se produjeron más de 2300 t; de esta manera, durante el periodo 2000-2014, la producción en la entidad registró una tasa de crecimiento promedio anual negativa de $1.63 \%$, la cual fue menor a la registrada a nivel nacional, situación que deja de manifiesto la importancia de la actividad en la entidad, no obstante que registra un ritmo lento, muestra una recuperación respecto al escenario nacional.

De la misma manera, durante el ciclo 2016, el Estado de México produjo $250 \mathrm{t}$ de café verde en una superficie de 475 ha; $67.39 \%$ de dicha producción se concentró en el Distrito de Desarrollo Rural (DDR) de Tejupilco, el cual por sus condiciones climáticas y orográficas posee las condiciones idóneas para la producción de café de calidad, los municipios que sobresalen en la producción del aromático son Amatepec, el cual participa con $73.82 \%$ de la producción y Temascaltepec $16.8 \%$.

El municipio de Amatepec cuenta con más de 300 productores que cultivan un total de 350 ha del aromático, donde se emplean a más de mil trabajadores en las labores de corte de la cereza principalmente; asimismo, los productores del municipio han conformado algunas empresas, las cuales comercializan el café procesado en diferentes presentaciones como café en grano, tostado y molido, de esta manera, al menos un integrante de cada familia del municipio se dedica al cultivo del café (Estrada, 2017).

En este sentido, la producción de dicho cultivo se ha constituido como una actividad importante en la agricultura de la región, representando una fuente importante en la generación de empleo e ingreso para la población de escasos recursos, así como un incentivo de crecimiento económico y comercial en la región sur del Estado de México. 
Bajo este contexto, el cultivo del café representa una actividad tradicional importante en la región sur del Estado de México; sin embargo, el impacto y la problemática de dicha actividad en la economía de la región ha sido poco abordado; en este sentido, resulta relevante, estudiar y analizar la situación que presenta la producción y comercialización del cultivo del café, a fin de detectar la principal problemática de la actividad en sus diferentes etapas, para posteriormente emitir juicios de opinión y alternativas de solución (Li et al., 2006; MacDonald, 2007).

\section{Materiales y métodos}

La investigación se llevó a cabo de abril a julio de 2018, periodo en el cual se recabó la información de campo; se realizó un muestreo por intención (Cochran, 1984), se encuestó a 41 productores de café de los municipios de Amatepec, Temascaltepec, Tlataya y Sultepec en el Estado de México, los cuales representan 50.61\% de los productores que conforman la Cooperativa Café Orgánico de Amatepec (CAFOA), un acopiador, 10 detallistas y 30 consumidores. El objetivo de las encuestas consistió en recabar información referente al proceso de producción, agentes participantes, costos de comercialización, volúmenes y precios corrientes de compra y venta, elementos que, para determinar los márgenes de comercialización, así como caracterizar la producción.

\section{Sistemas de cálculo}

Para calcular los márgenes de comercialización se cuenta con dos sistemas: el directo y el indirecto; el sistema más idóneo es el directo, el cual consiste en: a) seguir lotes estadísticamente representativos de los productos agrícolas, desde que salen de la finca hasta que llegan al consumidor final; b) registrar los costos y precios que se van originando en el trayecto del producto por los distintos agentes participantes; y c) delimitar la investigación a lotes representativos del movimiento de los productos, usando el muestreo estadístico para seleccionar los segmentos a estudiar, con el intención de que los resultados puedan considerarse como una estimación de los márgenes verdaderos (García et al., 1990).

El sistema de cálculo directo suministra información muy completa para el cálculo de los márgenes totales y sus componentes, sin embargo, el procedimiento es muy complicado y costoso. En la presente investigación se utilizó el método directo, ya que es más veraz y confiable respecto al cálculo y análisis de la información obtenida.

\section{Información utilizada}

Los datos referentes a los precios de compra y venta se obtuvieron de los agentes participantes en el proceso de comercialización; dicha información fue ponderada por los volúmenes de compra y venta del producto, con lo cual se calcularon los precios reales; el producto que se tomó como referencia para unificar la información y calcular los precios fue el café tostado molido.

\section{Procedimiento de estimación}

En la estimación de los márgenes de comercialización es de suma importancia vigilar que en todo el proceso la información utilizada sea comparable; es decir, que se refiera a la misma unidad y calidad de los productos, ya sean elaborados o no elaborados. 
De igual manera, en el proceso de comercialización del productor hacia el consumidor final se generan subproductos, por lo cual los precios que recibe el productor no son directamente comparables con los precios de venta al consumidor final. Para este caso, en el cálculo de los márgenes se tiene que resolver el problema de determinar el valor equivalente. De esta manera, el margen absoluto total de comercialización (M) se calcula por diferencia entre el valor del producto en consumo $(\mathrm{Pc})$ y el valor corregido en producción $(\mathrm{Pp})$ más los costos de comercialización en que se incurre durante el proceso (CC); es decir, $\mathrm{M}=\mathrm{Pc}-\mathrm{Pp}-\mathrm{CC}$.

En este sentido, un margen de comercialización se refiere a la diferencia entre el precio de venta de una unidad de producto por un agente de comercialización y el pago realizado en la compra de la cantidad de producto equivalente a la unidad vendida. Además, los márgenes están constituidos por una serie de componentes correspondientes a distintos costos y beneficios de los agentes, tales como el valor en pesos del trabajo utilizado, del transporte, de los materiales, envases y embalajes utilizados, la publicidad, la depreciación, los impuestos, los beneficios, intereses, alquileres y otros costos, los cuales se denominan costos de comercialización (CC) (García et al., 1990).

De esta manera, el margen bruto de comercialización (MBC), expresado en porcentaje, se define como la diferencia entre el precio por kilogramo que paga el consumidor final y el precio por kilogramo recibido por el productor. El análisis de los $\mathrm{MBC}$ tiene como objetivo determinar su magnitud en las distintas etapas de la comercialización, según el tipo de agente participante, así como la distribución del ingreso entre sus actores (Acuña, 1980; citado por Vilavoa et al., 2010); el $\mathrm{MBC}$ se calcula en relación con el precio final o precio pagado por el último consumidor y se expresa en términos porcentuales; así, el: $\mathrm{MBC}=[(\mathrm{PC}-\mathrm{PP}) / \mathrm{PC}] * 100$. Donde: $\mathrm{PC}=$ es el precio al consumidor; y $\mathrm{PP}=$ precio al productor (Mendoza, 1987).

\section{Costos de comercialización}

Para calcular los márgenes de comercialización se determinaron como componentes de los costos de comercialización (CC), en los cuales incurrieron los agentes participantes, a la mano de obra directa, los costos de acarreo, la energía eléctrica, el agua, la depreciación de la maquinaria y equipo, el almacenamiento, los gastos administrativos, la mano de obra indirecta y otros costos diversos.

\section{Consideración de los subproductos}

En el caso de los productos agrícolas y pecuarios, es importante abordar debidamente el aspecto concerniente a los subproductos obtenidos, con el fin de determinar su cantidad equivalente investigaciones realizadas por Muradian y Pelupessy (2005) afirman estos aspectos. Para el caso del café, el definir adecuadamente dicho valor es trascendente, ya que es comparable el valor del café cereza recibido por el productor, con el valor del café tostado y molido comprado por el consumidor final, debido a las prácticas de poscosecha (beneficio y secado) a que se somete el producto.

Una regla práctica para solucionar este problema puede consistir en corregir el precio al productor disminuyéndolo en el porcentaje representado por los subproductos (Renard, 1999). Este porcentaje puede ser en términos físicos o en términos de valor, mismo que se calcula en la fase en que se separan el producto principal y los subproductos. De acuerdo con esto, el margen de comercialización se calcula por diferencia entre el valor del producto en consumo y el valor corregido en producción. 
De esta manera, para calcular dicha cantidad equivalente, se utilizó al café tostado como unidad de medida equivalente, para lo cual se utilizó la medida de conversión propuesta por la Organización Internacional del Café (ICO) (2018), donde para convertir el café tostado en grano, a café verde equivalente, se multiplica el peso neto del café tostado por el factor 1.19, con lo cual se determinó el valor corregido en producción y permitió hacer comparables los precios del producto durante todo el proceso de comercialización y calcular los márgenes correspondientes (Pérez y Ruiz, 2017).

\section{Resultados y discusión}

\section{Sistema de producción}

Acorde con la clasificación de Moguel y Toledo (1996), la producción de café en la región sur del Estado de México se caracteriza por ser un sistema de producción rústico o de montaña, caracterizado por plantaciones de la especie arábiga, principalmente de las variedades typica y caturra, las cuales se desarrollan bajo un ambiente de sombra; la producción se desarrolla por pequeños productores dispersos en la región, cuyas superficies de siembra no superan las 3 ha, la mano de obra utilizada es esencialmente familiar, con contrato de jornales durante la época de cosecha; los beneficios de dicha actividad representan un ingreso complementario al de otras actividades pecuarias, agrícolas y de servicios desempeñadas por los productores.

\section{Agentes y canales de comercialización}

Los agentes participantes en el proceso de comercialización del café en la región sur del Estado de México son: los productores, un acopiador, constituido por la cooperativa de productores CAFOA, los detallistas y los consumidores finales.

El canal de comercialización tradicional que sigue el aromático desde su salida de la finca hasta su llegada al consumidor final es: productor $\rightarrow$ cooperativa CAFOA, el cual se realiza por $60 \%$ de los agentes participantes en el proceso.

Por su parte, $15 \%$ de los agentes practican la siguiente vertiente de dicho canal tradicional: productor $\rightarrow$ detallista $\rightarrow$ consumidor final y otro $15 \%$ siguen el canal: productor $\rightarrow$ consumidor final, mientras que el restante $10 \%$ de los productores no comercializan su producto, destinándolo al autoconsumo (Figura 1).

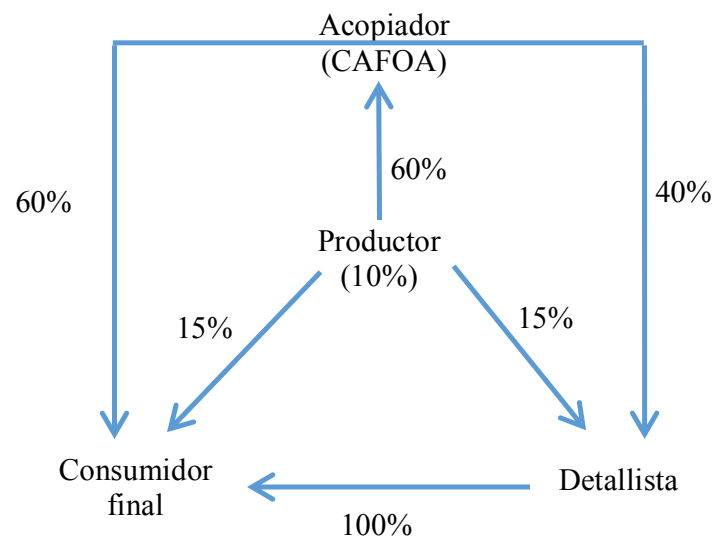

Figura 1. Cadena de comercialización del café. 


\section{Precios de venta}

En relación con los precios de venta alcanzados por los diferentes agentes participantes en el proceso de comercialización, durante el año 2018, sobresalen los detallistas, quienes registraron un precio promedio de venta de $162.92 \$ \mathrm{~kg}^{-1}$ de café tostado molido, el acopiador, constituido por la cooperativa CAFOA, con $148.26 \$ \mathrm{~kg}^{-1}$, mientras que los productores obtuvieron un precio de $122.91 \$ \mathrm{~kg}^{-1}$. Respecto a la participación en el precio de venta del producto, los detallistas contribuyeron con $8.92 \%$, el acopiador con $15.62 \%$ y los productores obtuvieron la mayor participación de $75.46 \%$ del precio final de venta.

El mayor precio de venta, alcanzado por los productores se registró en el mes de septiembre, el cual coincide con una menor oferta del producto, el acopiador por su parte, registro el mejor precio durante el periodo de enero a abril, mientras que los detallistas obtuvieron los mejores precios en los meses de julio, agosto y septiembre (Cuadro 1).

Cuadro 1. Participación de los diferentes agentes en el precio de venta.

\begin{tabular}{lcccccc}
\hline \multicolumn{1}{c}{ Mes/agente } & $\begin{array}{c}\text { Productor } \\
\left(\$ \mathrm{~kg}^{-1}\right)\end{array}$ & $\begin{array}{c}\text { Part } \\
(\%)\end{array}$ & $\begin{array}{c}\text { Acopiador } \\
\left(\$ \mathrm{~kg}^{-1}\right)\end{array}$ & $\begin{array}{c}\text { Part } \\
(\%)\end{array}$ & $\begin{array}{c}\text { Detallista } \\
\left(\$ \mathrm{~kg}^{-1}\right)\end{array}$ & $\begin{array}{c}\text { Part } \\
(\%)\end{array}$ \\
\hline Enero & 122.11 & 74.92 & 149.26 & 16.65 & 163.00 & 8.43 \\
Febrero & 115.96 & 76.15 & 149.26 & 21.87 & 152.28 & 1.98 \\
Marzo & 117.89 & 75.1 & 149.26 & 19.98 & 156.98 & 4.92 \\
Abril & 120.35 & 73.38 & 149.26 & 17.62 & 164.03 & 9 \\
Mayo & 122.29 & 74.22 & 148.79 & 16.09 & 164.76 & 9.69 \\
Junio & 125.10 & 74.86 & 148.79 & 14.17 & 167.12 & 10.97 \\
Julio & 126.55 & 75.71 & 147.41 & 12.48 & 167.14 & 11.81 \\
Agosto & 125.44 & 75.05 & 147.41 & 13.14 & 167.14 & 11.81 \\
Septiembre & 130.92 & 78.33 & 147.41 & 9.87 & 167.14 & 11.81 \\
Octubre & 118.58 & 71.74 & 147.41 & 17.44 & 165.29 & 10.82 \\
Noviembre & 126.61 & 80.3 & 147.41 & 13.19 & 157.67 & 6.51 \\
Diciembre & 123.14 & 75.76 & 147.41 & 14.93 & 162.54 & 9.31 \\
Promedio & 122.91 & 75.46 & 148.26 & 15.62 & 162.92 & 8.92 \\
\hline
\end{tabular}

\section{Margen bruto de comercialización}

Para calcular el margen bruto de comercialización (MBC) se toma en cuenta el precio final o precio pagado por el último consumidor y se expresa en términos porcentuales; de esta manera, el MBC mostró que por cada peso que pagó el consumidor final al adquirir un kilogramo de café tostado molido, los intermediarios obtuvieron $24.54 \%$ de dicho precio, esto es $40.01 \$ \mathrm{~kg}^{-1}$, por su parte, los productores consiguieron $75.46 \%$ del precio total pagado por el consumidor $\left(122.91 \$ \mathrm{~kg}^{-1}\right)$. Los intermediarios registraron los mejores márgenes brutos de comercialización durante los meses de junio, julio, agosto y septiembre (Cuadro 2). 
Cuadro 2. Margen bruto de comercialización.

\begin{tabular}{lccc}
\hline Mes/agente & $\begin{array}{c}\text { Productor } \\
\text { precio de venta }\left(\$ \mathrm{~kg}^{-1}\right)\end{array}$ & $\begin{array}{c}\text { Detallista } \\
\text { precio de venta }\left(\$ \mathrm{~kg}^{-1}\right)\end{array}$ & $\begin{array}{c}\text { Margen } \\
(\mathrm{MBC})(\%)\end{array}$ \\
\hline Enero & 122.11 & 163.00 & 25.08 \\
Febrero & 115.96 & 152.28 & 23.85 \\
Marzo & 117.89 & 156.98 & 24.9 \\
Abril & 120.35 & 164.03 & 26.62 \\
Mayo & 122.29 & 164.76 & 25.78 \\
Junio & 125.10 & 167.12 & 25.14 \\
Julio & 126.55 & 167.14 & 24.29 \\
Agosto & 125.44 & 167.14 & 24.95 \\
Septiembre & 130.92 & 167.14 & 21.67 \\
Octubre & 118.58 & 165.29 & 28.26 \\
Noviembre & 126.61 & 157.67 & 19.7 \\
Diciembre & 123.14 & 162.54 & 24.24 \\
Promedio & 122.91 & 162.92 & 24.54 \\
\hline
\end{tabular}

De la utilidad bruta total (MBC) registrada durante todo el proceso de intermediación (24.54\%), la cooperativa CAFOA alcanzó el mejor margen de comercialización, ya que por cada kilogramo de café tostado molido vendido obtuvo $\$ 25.34$ de utilidad (15.62\% de la utilidad bruta), el margen más alto se registró en el mes de febrero $\left(33.30 \$ \mathrm{~kg}^{-1}\right)$ y el más bajo en septiembre $\left(16.49 \$ \mathrm{~kg}^{-1}\right)$, por su parte, los detallistas alcanzaron un MBC de $14.67 \$ \mathrm{~kg}^{-1}$, que representa $8.92 \%$ de la utilidad bruta total, registrando en los meses de julio, agosto y septiembre el margen más alto con $19.73 \$$ $\mathrm{kg}^{-1}$ (Cuadro 3 y 4).

Cuadro 3. Márgenes brutos de comercialización en la intermediación.

\begin{tabular}{lccccc}
\hline $\begin{array}{l}\text { Acopiador a } \\
\text { detallista }\end{array}$ & $\begin{array}{c}\text { Precio al } \\
\text { acopiador }\end{array}$ & $\begin{array}{c}\text { Precio al } \\
\text { productor }\end{array}$ & $\begin{array}{c}\text { Precio al } \\
\text { consumidor final }\end{array}$ & $\begin{array}{c}\text { MBC } \\
(\%)\end{array}$ & $\begin{array}{c}\text { MBC } \\
\left(\$ \mathrm{~kg}^{-1}\right)\end{array}$ \\
\hline Enero & 149.26 & 122.11 & 163.00 & 16.65 & 27.15 \\
Febrero & 149.26 & 115.96 & 152.28 & 21.87 & 33.30 \\
Marzo & 149.26 & 117.89 & 156.98 & 19.98 & 31.36 \\
Abril & 149.26 & 120.35 & 164.03 & 17.62 & 28.91 \\
Mayo & 148.79 & 122.29 & 164.76 & 16.09 & 26.50 \\
Junio & 148.79 & 125.10 & 167.12 & 14.17 & 23.69 \\
Julio & 147.41 & 126.55 & 167.14 & 12.48 & 20.86 \\
Agosto & 147.41 & 125.44 & 167.14 & 13.14 & 21.97 \\
Septiembre & 147.41 & 130.92 & 167.14 & 9.87 & 16.49 \\
Octubre & 147.41 & 118.58 & 165.29 & 17.44 & 28.83 \\
Noviembre & 147.41 & 126.61 & 157.67 & 13.19 & 20.80 \\
Diciembre & 147.41 & 123.14 & 162.54 & 14.93 & 24.27 \\
Promedio & 148.26 & 122.91 & 162.92 & 15.62 & 25.34 \\
\hline
\end{tabular}


Cuadro 4. Márgenes brutos de comercialización en la intermediación.

\begin{tabular}{lccccc}
\hline $\begin{array}{l}\text { Detallista a } \\
\text { consumidor } \\
\text { final }\end{array}$ & $\begin{array}{c}\text { Precio al } \\
\text { consumidor }\end{array}$ & $\begin{array}{c}\text { Precio al } \\
\text { acopiador }\end{array}$ & $\begin{array}{c}\text { Precio al último } \\
\text { consumidor }\end{array}$ & $\begin{array}{c}\text { MBC } \\
(\%)\end{array}$ & $\begin{array}{c}\mathrm{MBC} \\
\left(\$ \mathrm{~kg}^{-1}\right)\end{array}$ \\
\hline Enero & 163.00 & 149.26 & 163.00 & 8.43 & 13.74 \\
Febrero & 152.28 & 149.26 & 152.28 & 1.98 & 3.02 \\
Marzo & 156.98 & 149.26 & 156.98 & 4.92 & 7.72 \\
Abril & 164.03 & 149.26 & 164.03 & 9 & 14.77 \\
Mayo & 164.76 & 148.79 & 164.76 & 9.69 & 15.97 \\
Junio & 167.12 & 148.79 & 167.12 & 10.97 & 18.33 \\
Julio & 167.14 & 147.41 & 167.14 & 11.81 & 19.73 \\
Agosto & 167.14 & 147.41 & 167.14 & 11.81 & 19.73 \\
Septiembre & 167.14 & 147.41 & 167.14 & 11.81 & 19.73 \\
Octubre & 165.29 & 147.41 & 165.29 & 10.82 & 17.88 \\
Noviembre & 157.67 & 147.41 & 157.67 & 6.51 & 10.26 \\
Diciembre & 162.54 & 147.41 & 162.54 & 9.31 & 15.13 \\
Promedio & 162.92 & 148.26 & 162.92 & 8.92 & 14.67 \\
Total & & & & 24.54 & 40.01 \\
\hline
\end{tabular}

\section{Márgenes totales de comercialización}

El margen total promedio registrado durante todo el proceso de comercialización del café en la región sur del Estado de México, fue de $36.6 \$ \mathrm{~kg}^{-1}$; la cooperativa de productores CAFOA, obtuvo el mayor margen con $23.95 \$ \mathrm{~kg}^{-1}$, mientras el restante $12.65 \$ \mathrm{~kg}^{-1}$ correspondió a los detallistas; los mayores márgenes totales (absolutos) de comercialización se alcanzaron en los meses de octubre y abril, con $43.3 \$ \mathrm{~kg}^{-1}$ y $40.26 \$ \mathrm{~kg}^{-1}$, respectivamente; por su parte, los márgenes totales más bajos se registraron en los meses de noviembre $\left(27.65 \$ \mathrm{~kg}^{-1}\right)$ y septiembre $\left(29.97 \$ \mathrm{~kg}^{-1}\right)$.

La cooperativa de productores CAFOA registró los mejores márgenes durante los meses de febrero (31.91 $\left.\$ \mathrm{~kg}^{-1}\right)$ y marzo $\left(61.22 \$ \mathrm{~kg}^{-1}\right)$, mientras que los más bajos fueron en septiembre $\left(15.10 \$ \mathrm{~kg}^{-1}\right)$ y noviembre $\left(19.41 \$ \mathrm{~kg}^{-1}\right)$, lo cual coincide con la mayor oferta del producto originado por el periodo de cosecha; por su parte, los detallistas alcanzaron mejores márgenes durante los meses de julio, agosto y septiembre con $17.71 \$ \mathrm{~kg}^{-1}$, lapso de menor oferta, y un menor margen durante febrero $\left(1.00 \$ \mathrm{~kg}^{-1}\right)$ y marzo $\left(5.70 \$ \mathrm{~kg}^{-1}\right)$.

Con el comportamiento anterior, queda de manifiesto la distribución de los ingresos que se generan entre los diferentes agentes participantes en el proceso de comercialización, así como el complemento que se genera con la oferta del producto (Cuadro 5). 
Cuadro 5. Márgenes totales de comercialización de los agentes participantes $\left(\$ \mathrm{~kg}^{-1}\right)$.

\begin{tabular}{|c|c|c|c|c|c|c|c|c|c|c|c|c|}
\hline \multirow{2}{*}{ Mes } & \multicolumn{4}{|c|}{$\begin{array}{c}\text { Margen } 1 \\
\text { productor a acopiador }\end{array}$} & \multicolumn{4}{|c|}{$\begin{array}{c}\text { Margen } 2 \\
\text { acopiador a detallista }\end{array}$} & \multicolumn{4}{|c|}{$\begin{array}{l}\text { Margen absoluto } \\
\text { detallista a productor }\end{array}$} \\
\hline & $\mathrm{PC}$ & $\mathrm{CC}$ & PV & $\mathrm{M}$ & $\mathrm{PC}$ & $\mathrm{CC}$ & PV & $\mathrm{M}$ & $\mathrm{PC}$ & $\mathrm{CC}$ & PV & M \\
\hline Enero & 122.11 & 1.39 & 149.26 & 25.76 & 149.26 & 2.02 & 163.00 & 11.72 & 122.11 & 3.41 & 163.00 & 37.48 \\
\hline Febrero & 15.96 & 1.39 & 149.26 & 31.91 & 149.26 & 2.02 & 152.28 & 1.00 & 115.96 & 3.41 & 152.28 & 32.91 \\
\hline Marzo & 117.89 & 1.39 & 149.26 & 29.97 & 149.26 & 2.02 & 156.98 & 5.70 & 117.89 & 3.41 & 156.98 & 35.67 \\
\hline Abril & 120.35 & 1.39 & 149.26 & 27.52 & 149.26 & 2.02 & 164.03 & 12.75 & 120.35 & 3.41 & 164.03 & 40.26 \\
\hline Mayo & 122.29 & 1.39 & 148.79 & 25.11 & 148.79 & 2.02 & 164.76 & 13.95 & 122.29 & 3.41 & 164.76 & 39.06 \\
\hline Junio & 125.10 & 1.39 & 148.79 & 22.30 & 148.79 & 2.02 & 167.12 & 16.31 & 125.10 & 3.41 & 167.12 & 38.61 \\
\hline Julio & 126.55 & 1.39 & 147.41 & 19.47 & 147.41 & 2.02 & 167.14 & 17.71 & 126.55 & 3.41 & 167.14 & 37.18 \\
\hline Agosto & 125.44 & 1.39 & 147.41 & 20.58 & 147.41 & 2.02 & 167.14 & 17.71 & 125.44 & 3.41 & 167.14 & 38.29 \\
\hline Septiembre & 130.92 & 1.39 & 147.41 & 15.10 & 147.41 & 2.02 & 167.14 & 17.71 & 130.92 & 3.41 & 167.14 & 32.82 \\
\hline Octubre & 118.58 & 1.39 & 147.41 & 27.44 & 147.41 & 2.02 & 165.29 & 15.86 & 118.58 & 3.41 & 165.29 & 43.30 \\
\hline Noviembre & 126.61 & 1.39 & 147.41 & 19.41 & 147.41 & 2.02 & 157.67 & 8.24 & 126.61 & 3.41 & 157.67 & 27.65 \\
\hline Diciembre & 123.14 & 1.39 & 147.41 & 22.88 & 147.41 & 2.02 & 162.54 & 13.11 & 123.14 & 3.41 & 162.54 & 35.99 \\
\hline Promedio & 122.91 & 1.39 & 148.26 & 23.95 & 148.26 & 2.02 & 162.92 & 12.65 & 122.91 & 3.41 & 162.92 & 36.60 \\
\hline
\end{tabular}

$\mathrm{M}=$ margen $; \mathrm{PC}=$ precio de compra; $\mathrm{PV}=$ precio de venta; $\mathrm{CC}=$ costos de comercialización.

\section{Conclusiones}

La producción de café en la región sur del Estado de México se desarrolla bajo un sistema de producción rústico o de montaña, caracterizado por plantaciones bajo sombra, de la especie arábiga, las explotaciones no superan las 3 ha de superficie, las cuales se encuentran dispersas en la región, la actividad se desarrolla como un complemento de otras actividades, en la cual se emplea primordialmente mano de obra familiar. El canal tradicional de comercialización para llevar el producto desde la finca hasta el consumidor final es la venta directa del productor a la cooperativa de productores Café Orgánico Amatepec (CAFOA), la cual opera como principal intermediario, otras variantes de dicho canal son la venta del productor al consumidor final y a los detallistas.

La comercialización del producto se realiza esencialmente mediante la presentación de café tostado molido, proceso que realizan los productores por cuenta propia o con el apoyo de la cooperativa de productores, fase que le proporciona valor agregado al producto. Bajo este esquema de comercialización, los productores obtuvieron una mayor participación en los precios de venta, los cuales alcanzaron el nivel más alto en los meses de menor oferta. En los márgenes de comercialización resultantes en el proceso de intermediación, la cooperativa de productores registró las mejores utilidades durante los meses de mayor oferta del aromático, originadas por los altos volúmenes de compra venta. 


\section{Literatura citada}

ASERCA. 1997. Apoyos y Servicios a la Comercialización Agropecuaria. El café en México; doscientos años de su producción. Rev. Claridades Agropecuarias. México, DF. 40 p.

ASERCA. 2002. Apoyos y Servicios a la Comercialización Agropecuaria. Café de México: hacia los mercados de calidad. Revista Claridades Agropecuarias. México, DF. 56 p.

CEFP. 2001. Centro de Estudios de las Finanzas Públicas. El mercado del café en México. Cámara de Diputados. H. Congreso de la Unión. Palacio Legislativo de San Lázaro México, DF. 33 p.

Cochran, W. G. 1984. Técnicas de muestreo. CECSA. México, DF. 513 p.

Cruz, L. A.; Cervantes, H. J.; Ramírez, G. A.; Sánchez, G. P.; Damián, H. M. A. y Ramírez, V. B. 2015. La etnoagronomía en la construcción de propuestas de desarrollo rural para comunidades campesinas. Ra Ximhai. 5(11):185-194.

Escamilla, P. E. 2016, Las variedades de café en México ante el desafío de la roya. Programa Mexicano del carbono, 4 Breves de políticas públicas. Huatusco, Veracruz, México. Boletín informativo. $10 \mathrm{p}$.

Escamilla, P. E. y Díaz, C. S. 2016. Sistemas de cultivo de café en México. CENACAFÉ, Huatusco, Veracruz. $61 \mathrm{p}$.

Estrada, M. 2017. Café de Amatepec, una tradición en Edomex. Periódico Agencia Quadratín. https://edomex.quadratin.com.mx/cafe-amatepec-una-tradicion-edomex/.

García, M. R.; García, D. G. y Montero, H. R. 1990. Notas sobre mercados y comercialización de productos agrícolas. Centro de economía. Colegio de Postgraduados en Ciencias Agrícolas. Montecillo, Estado de México. 473 p.

González, J. A. 2004. Dealing with risk: small-scale coffee production systems in Mexico. Perspectivas Latinoamericanas. 1(22):1-39.

ICO 2014. International Coffee Organization. Estadísticas de producción y exportación de café. http://www.ico.org.

ICO. 2018. International Coffee Organization. Coeficientes de conversión del café. http://www.ico.org.

INIFAP. 2013. Instituto Nacional de Investigaciones Forestales, Agrícolas y Pecuarias. Cafeticultura en la zona centro del estado de Veracruz, diagnóstico, productividad y servicios ambientales. Centro de investigación regional Golfo Centro, Teocelo, Veracruz. $36 \mathrm{p}$.

Leme, P. H. and Machado, R. T. 2013. The quality pillars of a certification process: the coffee quality program (CQP) in Brazil. Agroalimentaria. 19(37):61-74.

Li, S.; Ragu-Nathan, B.; Ragu-Nathan, T. S. and Rao, S. S. 2006. The impact of supply chain management practices on competitive advantage and organizational performance. Omega. 34(2):107-124.

MacDonald, K. 2007. Globalizing justice within coffee supply chains? Fair trade, Starbucks and the transformation of supply chain governance. Third World Q. 28(4):793-812.

Mendoza, G. 1987. Compendio de mercadeo de productos agropecuarios. Instituto Interamericano de Cooperación para la Agricultura. San José, Costa Rica. 343 p.

Moguel, P. y Toledo, V. M. 1996. El café en México, ecología, cultura indígena y sustentabilidad. Rev. Cienc. 43(2):40-51.

Muradian, R. and Pelupessy, W. 2005. Governing the coffee Chain: the role of voluntary regulatory Systems. World Dev. 33(12):2029-2044. 
Pérez, F. Y. y Ruiz, E. F. 2017. Construyendo autogestión en la producción y conservación del café de calidad en Teocelo, Veracruz. Universidad Autónoma Chapingo (UACH)-Centro Regional Universitario del Anáhuac. Primera (Ed.). Chapingo, Texcoco, Estado de México. $61 \mathrm{p}$.

Renard, M. C. 1999. The interstices of globalization: the example of fair coffee. Sociol. Rural. 39(4):484-500.

SAGARPA. 2017. Secretaría de Agricultura, Ganadería, Desarrollo Rural, Pesca y Alimentación. Sistema de Información Agroalimentaria de Consulta (SIACON). http://www.siap.gob.mx.

Vilaboa, A. J.; Díaz, R. P.; Platas, R. D. E.; Ruiz, R. O.; González, M. S. S. y Juárez, L. F. 2010. Fallas de mercado y márgenes de comercialización en bovinos destinados al abasto de carne en la región del Papaloapan, Veracruz. Rev. Econ. Soc. Territ. X(34):813-833. 